American Journal of Immunology 2 (1): 19-22, 2006

ISSN 1553-619X

(c) 2006 Science Publications

\title{
Polymyositis Initiation Involving Amlodipine Besylate
}

\author{
${ }^{1}$ R.M. Golding, ${ }^{2}$ L.G.F. Giles and ${ }^{3}$ E.M. Sokoya \\ ${ }^{1}$ The University of New South Wales, Sydney, New South Wales 2052, Australia \\ ${ }^{2}$ Murdoch University, South Street, Murdoch, Western Australia 6150, Australia and James Cook \\ University of North Queensland, Townsville, Queensland 4810, Australia \\ ${ }^{3}$ Department of Anesthesiology, Baylor College of Medicine, Houston, TX 77030, USA
}

\begin{abstract}
Based on extensive blood pressure data and results from a scientific model of the analysis of the rate processes involved in polymyositis the evidence suggests that the polymyositis in a particular patient was initiated by taking the drug amlodipine besylate (norvasc). The method of our analysis should serve as a foundation in handling other drug related interactions.
\end{abstract}

Key words: Polymyositis, blood pressure, amlodipine besylate (norvasc), steroid therapy

\section{INTRODUCTION}

On revisiting an extensive blood pressure database of a specific polymyositis patient prior to the diagnosis of the disease, we have been able to identify when the body began producing the chemicals that attacked the muscles. Furthermore, the evidence suggests that the process was triggered when the patient began to take amlodipine besylate (norvasc) in an attempt to reduce the blood pressure.

For this particular patient, considerable detail is given by Golding ${ }^{[1]}$ and extends the mathematical model developed earlier ${ }^{[2,3]}$ to control and monitor polymyositis. Golding and Giles ${ }^{[4]}$ confirm a significant reduction in the muscle inflammation observed from two muscle biopsies spanning eight years, illustrating the success of the model.

For almost five years prior to the diagnosis of polymyositis, blood pressure data was gathered specifically as a matter of general interest. About two years prior to the diagnosis the patient commenced taking norvasc. Prior to that, the patient took no drugs. In this study we present evidence that suggests that it was the norvasc that initiated the onset of polymyositis.

The data measurements: First, we shall consider the blood pressure data over a period of 1772 days prior to the introduction of prednisolone. We shall find it convenient to group the data into two sets, set A and set $B$.

Set A: from -1772 to -795 days.

In set $\mathrm{A}$, the blood pressure measurements were not taken daily and represent 69 specific readings spread out over the period.

Set B: from -793 to -1 days.

In set $\mathrm{B}$, although the blood pressure measurements also were not taken daily they were recorded more frequently than in set A, especially when the norvasc was commenced and varied. In set B we have 147 specific readings spread out over the period. The mean and standard deviations of the blood pressure data over the two sets are given in Tables 1 and 2 .

Next we may compare the results using the Student's t-test $\mathrm{p}$-value ${ }^{[5]}$ (Table 3 ). If the $\mathrm{p}$-value is greater than 0.995 then the distributions are significantly different. For the comparison between set $A$ and set $B$, the only value that has not been affected is the pulse pressure. Hence, over the entire period of 1772 days, the mean pulse pressure was $50.333 \mathrm{mmHg}$ with a standard deviation of $7.0720 \mathrm{mmHg}$. To illustrate the change between sets A and B data, we have shown the two heart rate distributions in Fig. 1.

Figure 1 highlights that an abrupt change in the heart rate took place following day -795 . This coincides with the day that the patient started taking norvasc. From day -795 to day -392 days, $5 \mathrm{mg}$ or 10 $\mathrm{mg}$ norvasc was used. From day -315 until the prednisolone was introduced, 0.5 or $0.1 \mathrm{mg}$ trandolapril (odrik) was used. During these two periods, norvasc and or, odrik was used and varied for short periods of time. We shall choose the periods when only norvasc was used as set $\mathrm{C}$ where the data is given in Table 4. We shall choose the periods when only odrik was used as set $\mathrm{D}$ where the data is given in Table 5 .

From Table 3, by comparing the distributions of sets $\mathrm{A}$ and $\mathrm{C}$, we establish that taking norvasc changes significantly all the blood pressure parameters from prior to taking norvasc. In the case of replacing the norvasc with odrik only, comparing the distributions of sets $\mathrm{A}$ and $\mathrm{D}$, the diastolic and the heart rate are significantly changed. Furthermore, by comparing the distributions of set $\mathrm{C}$ and set $\mathrm{D}$ it follows that the evidence is that all the blood pressure parameters have been significantly changed by replacing norvasc with odrik except for the heart rate.

In summary, it is clear that norvasc and odrik significantly affect the blood pressure results. It appears that the replacement of norvasc with odrik does not 
change the increase of the heart rate from the initial period designated as set $\mathrm{A}$. On the other hand, however, the other blood pressure characteristics are norvasc/odrik related and also differ from set A.

\section{ANALYSIS OF THE RESULTS}

Prior to day - 795 the patient had not taken any drugs. On day -795 the patient commenced taking $5 \mathrm{mg}$ per day of norvasc that was suggested in order to reduce the blood pressure values. At that point there was a very rapid change in the systolic, diastolic and heart rate values. In particular, the increase of the heart rate was very significant.

From Golding ${ }^{[1]}$, we may obtain an estimate, using steady state conditions, of the increase of the heart rate for our particular patient if it was due to chemicals attacking the muscles. We have shown ${ }^{[2]}$ that if the prednisolone is taken every second day the heart rate is different on day 1 and day 2 some 12 hours and 36 hours after taking the prednisolone. If we consider the $25 \mathrm{mg}, 50 \mathrm{mg}$ and $75 \mathrm{mg}$ cases with $2 \mathrm{mg}$ of odrik the heart rate on day 2 may be approximated as the same in all cases, namely, 80.1 per min from 210 data values with a standard deviation of 10.89 per min. For day 1 the mean heart rate values for the 25,50 and $75 \mathrm{mg}$ cases are 82.8 per min, 89.1 per min and 91.6 per min respectively. These values may be expressed approximately as heart rate $=79.0+0.18 \mathrm{P}$ where $\mathrm{P}$ is the predisolone concentration in $\mathrm{mg}$. In the case of no prednisolne we have a value of 79.0 per min which is close to the common value for day 2. Furthermore, this value gives us an estimation of the heart rate value due to the chemicals attacking the muscles that give rise to the inflammation. From Table 2 we observed a heart rate value of 79.0 per min following the intake of norvasc. Since day 2 is approximately independent of the prednisolone concentration over that range and is close to this value it implies that as far as the heart rate is concerned it appears to be constant and effectively related to the maximum level of the chemicals attacking the muscles.

Another approach is to examine the effect of a much higher prednisolone intake over a few days, where the average prednisolone concentration is very small and where the removal of the chemicals affecting the heart rate on the other hand is high. This is illustrated in Fig. 2 when prednisolone was initially used at $60 \mathrm{mg}$ per day for six days with $0.1 \mathrm{mg}$ per day of odrik. In this case, the observed heart rate decreased from about 85 per min to about 70 per min, the lower value in Fig. 1. We estimate that the average prednisolone concentration over that short period was about 0.24 to $0.35 \mathrm{mg}$, well below the value for the natural decay of about $8.57 \mathrm{mg}$.

Prior to the introduction of prednisolone and using only $0.1 \mathrm{mg}$ per day of odrik the mean heart rate over a 220-day period was 85.4 per min.

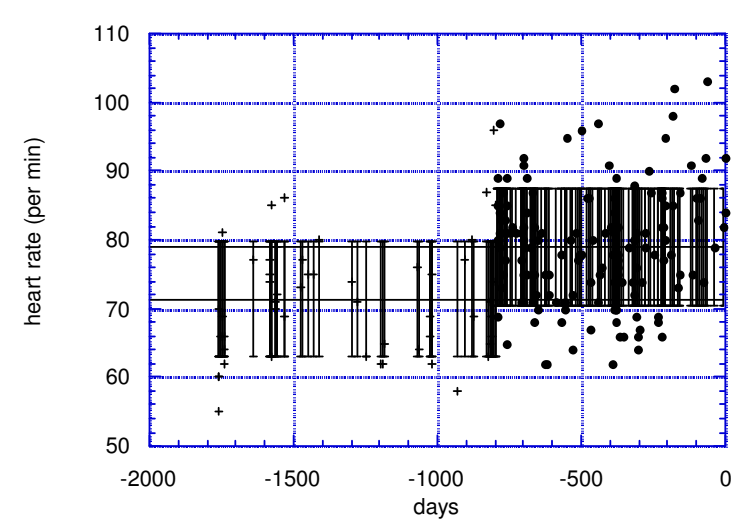

Fig.1: The heart rate 1772 days prior to the prednisolone intake. The standard deviations of the two distributions between the data in sets $\mathrm{A}$ and $\mathrm{B}$ are shown. Note that the abrupt change in the heart rate following day -795 coincide with the day that the patient started taking norvasc

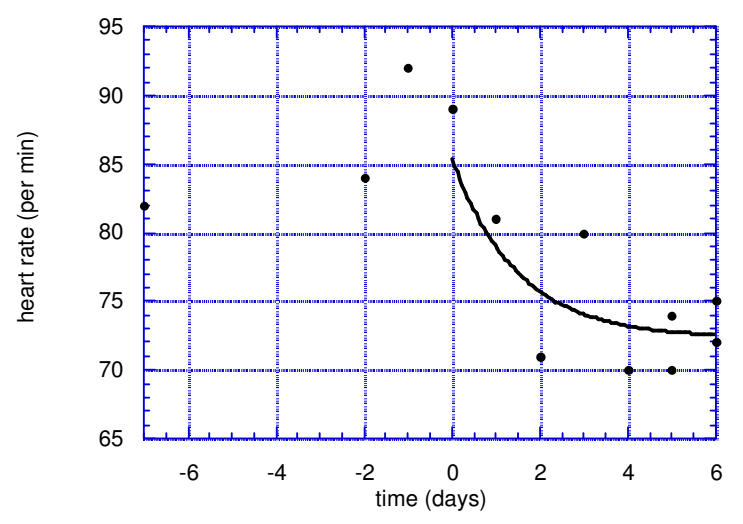

Fig. 2: The observed heart rate seven days before and six days after the intake of $60 \mathrm{mg}$ of prednisolone per day

These results reflect the processes taking place as defined in the model. Furthermore, it is clear that the chemicals interacting with the muscle yielding muscle inflammation increases the heart rate corresponding to that observed in Fig. 1. Secondly, the prednisolone level used to control the muscle inflammation also increases the heart rate. In Fig. 2 the introduction of a high concentration of prednisolone indicates a reduction in the heart rate within a few days. On day 7 the prednisolone intake was reduced to $50 \mathrm{mg}$ per day. The heart rate remained low for a few days before increasing to about 90 per min indicating that the prednisolone concentration was now affecting the heart rate.

Hence at a very high prednisolone concentration we observe two effects on the heart rate, namely, (i) the impact of the prednisolone and (ii) the prednisolone reducing significantly the chemicals giving rise to the inflammation. On the other hand at a moderate prednisolone concentration we observe only one effect on the heart rate, namely, (i) the impact of the prednisolone. 
Am. J. Immunology 2 (1): 19-22, 2006

Table 1: The summary of the data for set A i.e. for the period -1772 to -795 days

\begin{tabular}{lcccc}
\hline set A & Systolic $\mathrm{mmHg}$ & Diastolic $\mathrm{mmHg}$ & Pulse pressure mmHg & Heart rate per minute \\
\hline data points & 69 & 69 & 69 & 54 \\
mean & 142.41 & 89.406 & 53.000 & 71.370 \\
standard deviation & 8.5532 & 5.9685 & 6.387 & 8.4190 \\
\hline
\end{tabular}

Table 2: The summary of the data for set B i.e. for the period -793 to -1 days

\begin{tabular}{lcccc}
\hline set B & Systolic $\mathrm{mmHg}$ & Diastolic $\mathrm{mmHg}$ & Pulse pressure mmHg & Heart rate per minute \\
\hline data points & 147 & 147 & 147 & 147 \\
mean & 132.62 & 82.993 & 49.626 & 79.034 \\
standard deviation & 11.399 & 6.8502 & 7.1970 & 8.4606 \\
\hline
\end{tabular}

Table 3: The Student's t test p-value comparing several distributions

\begin{tabular}{lllll}
\hline & Systolic $\mathrm{mmHg}$ & Diastolic $\mathrm{mmHg}$ & Pulse pressure $\mathrm{mmHg}$ & Heart rate per minute \\
\hline A-B & 1.000 & 1.000 & 0.957 & 1.000 \\
A-C & 1.000 & 1.000 & 1.000 & 1.000 \\
A-D & 0.992 & 0.999 & 0.701 & 1.000 \\
B-C & 0.992 & 0.974 & 0.975 & 0.751 \\
B-D & 0.998 & 0.992 & 0.985 & 0.914 \\
C-D & 1.000 & 1.000 & 1.000 & 0.964 \\
\hline
\end{tabular}

Table 4: The summary of the blood pressure data over the period when only $5 \mathrm{mg}$ or $10 \mathrm{mg}$ of norvasc was taken each day

\begin{tabular}{lcccc}
\hline set $\mathrm{C}$ & Systolic $\mathrm{mmHg}$ & Diastolic $\mathrm{mmHg}$ & Pulse pressure mmHg & Heart rate per minute \\
\hline data points & 91 & 91 & 91 & 91 \\
mean & 128.97 & 81.187 & 47.780 & 78.286 \\
standard deviation & 10.674 & 6.922 & 6.588 & 7.799 \\
\hline
\end{tabular}

Table 5: The summary of the blood pressure data over the period when only $0.5 \mathrm{mg}$ or $0.1 \mathrm{mg}$ of odrik was taken each day

\begin{tabular}{lcccc}
\hline set D & Systolic $\mathrm{mmHg}$ & Diastolic $\mathrm{mmHg}$ & Pulse pressure $\mathrm{mmHg}$ & Heart rate per minute \\
\hline data points & 45 & 45 & 45 & 45 \\
mean & 138.07 & 85.756 & 52.311 & 81.089 \\
standard deviation & 10.001 & 5.621 & 7.245 & 9.591 \\
\hline
\end{tabular}

The results from Figs. 1 and 2 thus reinforce that the increase in the heart rate, following the introduction of norvasc, corresponds to the production of chemicals that effect the heart rate and may be radically reduced by using prednisolone under controlled conditions. Moreover, these results give an insight, previously unavailable in developing the model, of the likely time dependence of the chemicals affecting the muscles associated with the prednisolone interactions. (The model was primarily developed to handle steady state conditions and focussed on the processes associated with the chemicals that affected the muscles and not the heart rate, although they will be correlated.)

In a previous study ${ }^{[4]}$ a method for extracting muscle information from serum creatine kinase measurements flagged that the particular patient began having difficulty in climbing stairs and getting up from a chair which became increasingly difficult, with occasional collapses. This commenced after day -793 and reinforces the suggestion that a muscle problem began within 2 days after the first intake of norvasc.

Furthermore, a serum creatine kinase value of 949 IU/L was measured in the patient on day -355 . (Note that norvasc was still being taken.) It was not until after day -40 , following a muscle biopsy, that the patient was diagnosed as having polymyositis with chronic muscle inflammation. This was supported by the observation, over the period, of muscle wastage, in particular within the upper left leg (thigh) ${ }^{[4]}$.
Hence we have shown that the rapid significant increase in the heart rate may be explained very simply as due to the chemicals arising from polymyositis, where the rate of production of the chemicals occurred very rapidly on the introduction of norvasc. We may show also from the rate steps involving the chemicals attacking the muscles, that the release of additional creatine kinase by the muscles would rapidly increase to over $90 \%$ of the maximum value within four days. This would reflect what the patient experienced. After a year norvasc was replaced by trandolapril (odrik). We note that a detailed analysis of the blood pressure data show no justification in giving the patient norvasc and the diagnosis that the patient had Buerger's disease (thromboangiitis obliterans) that involves both arteries and veins, especially those of the lower extremities, has never been supported. Next we shall assess the information available in an attempt to quantify what has occurred.

First we shall address the change in the heart rate. Most publications report that norvasc has no effect on the heart rate $\mathrm{e}^{[6-10]}$. In a few cases norvasc has increased slightly the heart rate ${ }^{[11]}$ and in one case ${ }^{[12]}$ the increase was about 4 beats per min. There appears to be no previous publication that shows any increase that even comes close to what we have observed and measured.

In a recent study ${ }^{[4]}$ we outlined for a particular patient the monitoring and management of 
inflammatory disease and the likely processes taking place. A muscle biopsy, following almost two years after the norvasc intake was commenced, and over which period muscle difficulties were increasing, as supported by our heart rate analysis showed that the severe muscle inflammation was due to polymyositis. The second muscle biopsy, some eight years later, showed a major change with no muscle inflammation characterised. However, ragged red fibres were observed. Ragged red fibres due to mitochondrial changes are commonly found in the later stages of inclusion body myositis ${ }^{[13]}$. This observation would support the case as discussed in $^{[4]}$ that the muscle inflammation appears to be generated by two processes as quantified in ${ }^{[4]}$ as process (a) and process (b). If process (c) was involved, muscle inflammation would have been observed. Furthermore, there is no indication that the rates of the chemicals attacking the muscles have changed over the years. The reduction in the muscle attack, estimated to be about $90 \%$, is due to the prednisolone interaction with the chemicals that cause the muscle inflammation.

Norvasc is a $\mathrm{Ca}^{2+}$ channel blocker used for mild to moderate essential hypertension and for the treatment of angina pectoris ie. norvasc is a calcium ion influx inhibitor (slow channel blocker or calcium ion antagonist) that inhibits the transmembrane influx of calcium ions into cardiac and smooth muscle without changing serum calcium concentrations; the mechanism of the antihypertensive action of norvasc is due to a direct relaxant effect on vascular smooth muscle (MIMS Desk Reference 2004). After oral administration of therapeutic doses, norvasc is absorbed with peak blood levels between 6-12 hours post dose. According to MIMS (MIMS Desk Reference 2004), less common side-effects of norvasc are muscle cramps and myalgia. Pfizer found that most adverse reactions were of mild or moderate severity in controlled clinical trials directly comparing norvasc $(n=1730)$, in doses up to $10 \mathrm{mg}$, to placebo $(\mathrm{n}=1250)$; muscle cramps occurred in less than $1 \%$ in placebo controlled trials.

\section{CONCLUSION}

In summary, at and following the introduction of norvasc, the heart rate increased significantly. The increase corresponds to the observed heart rate measurements at steady state when the prednisolone concentration is much lower than the value used to effectively control the polymyositis. Secondly, the heart rate prior to the norvasc intake was obtained by using a high prednisolone intake for a few days. All the evidence underpins the suggestion that norvasc almost instantaneously triggered the rate of production, in our specific patient, of the chemicals that characterise the muscle attack leading to chronic muscle inflammation. Previous work ${ }^{[2]}$ has shown that the rate of production of these chemicals has not changed at least since the diagnosis of polymyositis.

Finally, this work illustrates the dangers of administering any drug without an understanding of the likely process or processes that may occur and the lack of knowledge to control and monitor the processes in a scientific way from appropriate detailed data.

\section{REFERENCES}

1. Golding, R.M., 2003. Polymyositis - a further investigation. Physiol. Meas., 24: 859-878.

2. Golding, E.M. and R.M. Golding, 2001. An insight into cortisol and polymyositis control with steroid therapy. Med. Hypoth., 57: 76-86.

3. Golding, E.M. and R.M. Golding, 2002. Controlling and monitoring polymyositis under steroid therapy. Med Hypoth., 59; 674-681.

4. Golding, R.M. and L.G.F. Giles, 2006. A method for extracting muscle information from serum creatine kinase measurements - its potential value in the monitoring and management of inflammatory muscle disease. Med. Hypoth., 66: 476-485.

5. Spiegel, M.R., 1980. Shaum's outline series of theory and problems of Probability and Statistics, McGraw-Hill.

6. Bilge, A.K., D. Atilgan, T. Tukek, M. Ozcan, B. Ozbeb, N. Kovlan and M. Meric, 2005. Effects of amlodipine and fosinopril on heart rate variability and left ventricular mass in mild-to-moderate essential hypertension. J Clin Pract., 59: 306.

7. Ohutsuka, S., A. Yamazaki, Y. Ovake, and I. Yamaguchi, 2003. Amlodipine improves vascular function in patients with moderate to severe hypertension. J Cardiovasc Pharmacol., 42: 296.

8. Islim, I.F., R.D. Watson, H.N. Ihenacho, M. Ebanks and S.P. Singh, 2001. Cardiology., 96 Suppl 1, 10.

9. Zanchetti, A., S. Omboni, P. La Commare, R. De Cesaris and P. Palatini, 2001. Efficacy, tolerability, and impact on quality of life of long-term treatment with manidipine or amlodipine in patients with essential hypertension. J. Cardiovasc Pharmacol., 38: 642-650.

10. Zaliunas, R., J. Brazdzionvte, V. Zabiela and R. Jurkevicius, 2005. Effects of amlodipine and lacidipine on heart rate variability in hypertensive patients with stable angina pectoris and isolated left ventricular diastolic dysfunction. Int $\mathbf{J}$ Cardiol., 101: 347

11. Palatini, P., A. Mugellini, V. Spagnuolo, M. Santonastaso, G.B. Ambrosia, A. Caiazza and E. Malacco, 2004. Comparison of the effects on 24-h ambulatory blood pressure of valsartan and amlodipine, alone or in combination with a lowdose diuretic, in elderly patients with isolated systolic hypertension (Valsyst Study). Blood Press Monit., 9: 91-7.

12. Kuramoto, K., S. Ichikawa, A. Hirai, S. Kanada, T. Nakachi and T. Ogihara, 2003. Azelnidipine and amlodipine: a comparison of their pharmacokinetics and effects on ambulatory blood pressure. Hypertens Res., 26: 201.

13. Oldfors, A., A.-R. Moslemi, L. Jonasson, M. Ohlsson, G. Kollberg and C. Linderberg, 2006. Mitochondrial abnormalities in inclusion-body myositis, Neurology, 66(Suppl 1) S49-S55. 